\title{
B2MML AS AN EXCHANGE FORMAT FOR ASSET ADMINISTRATION SHELLS AS PART OF A PLUG-AND-PRODUCE PROCESS FOR A FLUID POWER ENGINEERING APPLICATION
}

\author{
Hartmut Schweizer ${ }^{1 *}$, Raphael Alt ${ }^{2}$, Katharina Schmitz ${ }^{2}$, Martin Wollschlaeger ${ }^{1}$ \\ ${ }^{1}$ Institute for Applied Computer Science, TU Dresden, Nöthnitzer Str. 43, 01187 Dresden \\ ${ }^{2}$ Institute for Fluid Power Drives and Systems, RWTH-Aachen University, Campus-Boulevard 30, 52074 Aachen \\ * Corresponding author: Tel.: +49 351463 38068; E-mail address: hartmut.schweizer@tu-dresden.de
}

\begin{abstract}
One development in the course of industrial digitalisation, which is being driven forward particularly in the German-speaking countries and will gain in importance in the future, is that of the Asset Administration Shell (AAS) [1]. The present work deals with the distribution, instantiation and use of those AASs for assets within the domain of a fluid power engineering application, which by themselves do not currently have the corresponding hardware and software for harbouring an AAS. The Business to Manufacturing Markup Language (B2MML) is used here for the deployment and instantiation of these AASs. In the present work, B2MML is extended with the concept definitions of AAS using its enhancement mechanisms. The distribution of the AASs to computing resources then takes place using B2MML and its transaction definitions. Furthermore, B2MML is used in a submodel to orchestrate process queues. In the course of the FL4 research project, B2MML is integrated as a partial model into the AAS and is used for orchestrating the Plug-and-Produce processes one the business and on subordinate levels.
\end{abstract}

Keywords: Asset Administration Shell, B2MML, Automation

\section{INTRODUCTION}

The aim of the research project FL4 is a proof of concept for plug-and-produce in the field of fluid power by realizing an application based on industry 4.0 (I4.0) compliant components. For this purpose, a demonstrator consisting of two sets of components (hydraulics and pneumatics) is assembled.

The hydraulic application represents a positioning task under the influence of an external load. The hydraulic system consists of two modules, a constant pressure supply and a valve-controlled linear drive, which is tensioned by another valve-controlled linear drive. The pneumatic application is a 2D handling system for repositioning an object. The system consists of a pneumatic compressed air supply and a valve terminal with 5/3-way switching valves which actuate a gripper and two cylinders $(\mathrm{X}$ and $\mathrm{Z}$ direction) (Fig. 1).

Scope of the project is to proof interoperability of $\mathrm{I} 4.0$ compliant fluid components during commissioning. For this purpose, the administration shells of the individual components are to be implemented in such a way that they function in the form of a service-oriented architecture (SOA) and partially und fully automate the commissioning of the plant together.

For this reason, both applications are brought together on a demo platform and are put into operation by the same commissioning logic and personnel. In addition, the systems were designed in such a way that an exchange and recommissioning of another actuator or valve can be carried out simply by switching over at the test bench. This simulates the replacement of one component with another, thus demonstrating that the commissioning of all fluid components is supported equally. For the realization of an industry 4.0 compliant PnP scenario, a virtual representation was created for the demonstrator using several Raspberry Pies (RP). Each Raspberry Pi hosts the AAS of one component (Fig. 1).

Since in the present case many fluid power components are non-smart components whose 


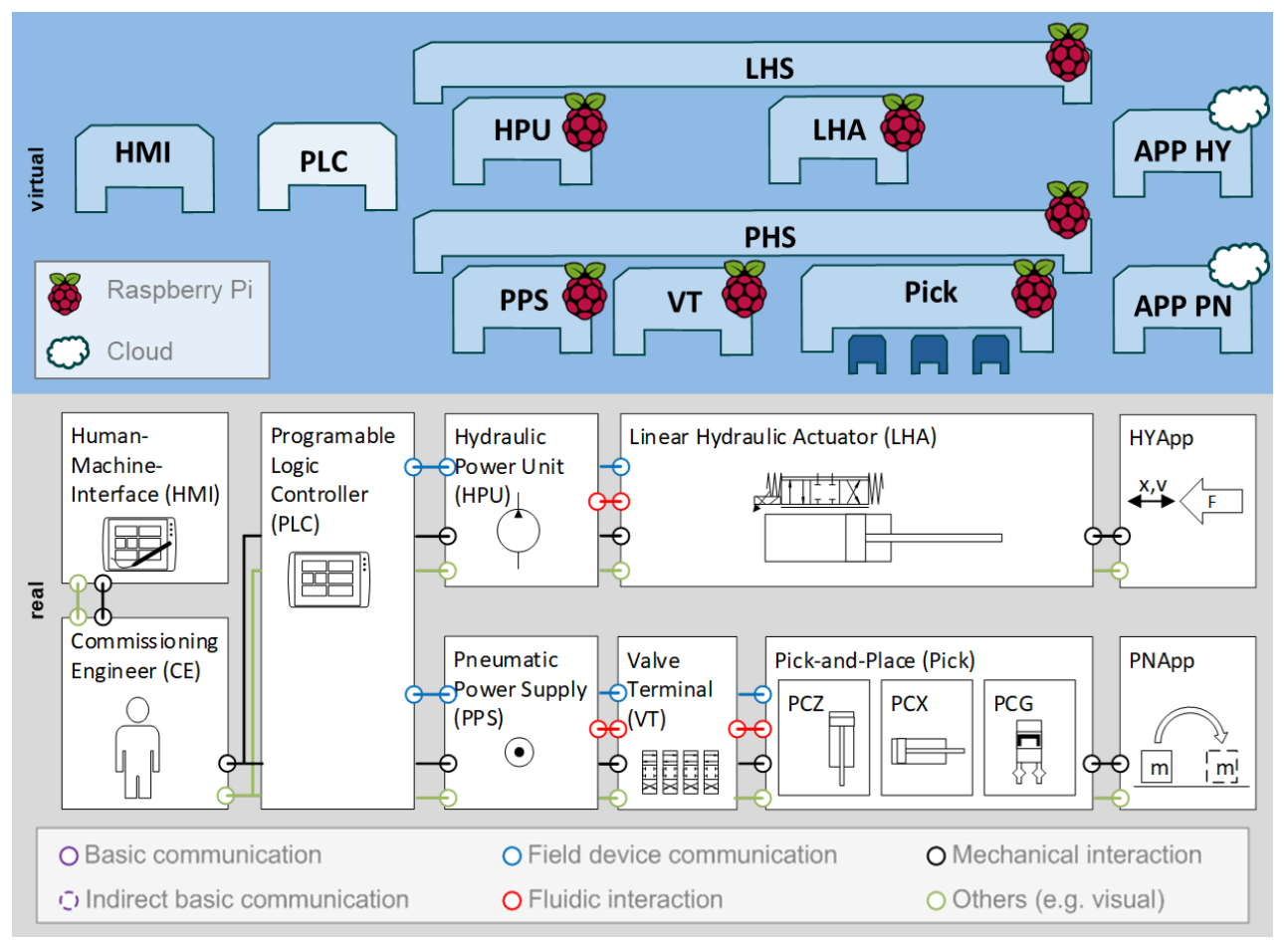

Figure 1: FL4 demonstrator (hydraulic and pneumatic components) with main virtual representation

AASs are not instantiated on the component itself, but on separate RPs, a standardized method is required to manage the deployment and exchange of the AASs. The present concept demonstrates an ERP-controlled approach using the IEC 62264 B2MML implementation for this processes. Furthermore, for partially and fully automated commissioning, the corresponding commissioning steps must be orchestrated. A solution approach with B2MML is also presented for this scenario. The runtime environment necessary therefor is currently being developed in form of a process engine for B2MML.

\section{TECHNOLOGIES}

In the following, the technologies of the Asset Administration Shell and the IEC 62264 implementation $\mathrm{B} 2 \mathrm{mml}$ used in the present concept are briefly explained.

\subsection{Asset Administration Shell}

The AAS represents a decisive development in the field of automation. As a digital representative of an asset, it exposes its features in form of properties. The AAS integrates the physical object into Industry 4.0 communication, is addressable in the network and uniquely identifies the asset. It allows finegrained access to all information of the object and provides standardized and secure communication interfaces.

It holds digital models of various aspects, so called submodels, and describes technical functionality exposed by the Administration Shell or respective assets (Fig. 2) [2].

A distinction can be made between passive and active AASs [11]. Passive AASs are data records in stored files are in databases (type 1), active AASs are instantiated and communicate with other AASs with properties in the form of functionality (type 2). The present work deals with the deployment and instantiation of AASs supporting a $\mathrm{PnP}$ procedure for fluid power 


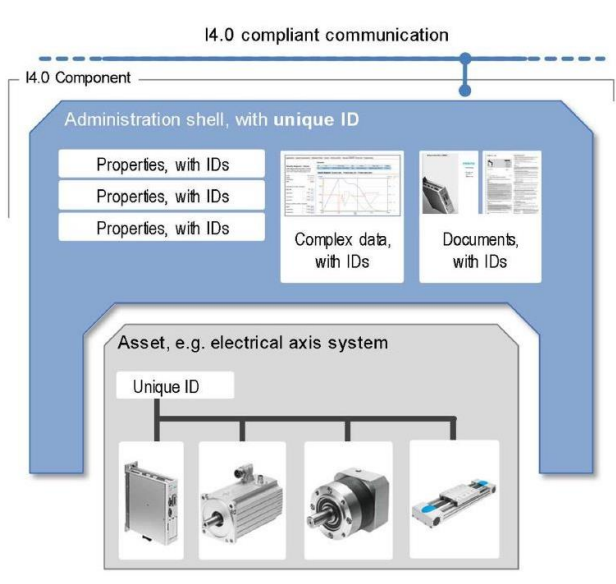

Figure 2: Asset Administration Shell [2]

applications and so AASs from type 2, e.g. a hydraulic power unit. This method is demonstrated with administration shell XML implementations, corresponding to the definition published in "The Asset Administration Shell in detail” (ZVEI) [2].

\subsection{B2MML}

The international standard IEC 62264 defines models, activities, and data exchange for Manufacturing Operations Management systems [5][7][8]. B2MML is an XML implementation of the IEC 62264 specifications in the form of XML schemas, which is designed as a link between ERP and materials management (supply chain) and to the production level [6]. Part 2 of IEC 62264 [7] defines objects and attributes for the integration of enterprise management and control systems. This includes the resource types Personnel, Equipment, Material, and Physical Asset as well as the production process type ProcessSegment. In the following, the equipment model, the process segment model and the transaction model (IEC 62264 Part 5 [8]) for the resources are explained in more detail.

\section{The role-based equipment model of IEC 62264}

Each Equipment resource can be composed of other Equipment resources and aggregates Equipment Properties. It can also be defined using one or more Equipment Classes, which can have any number of their own class properties. Test specifications can also be defined for Equipment and Equipment Classes and their properties. Equivalent models are defined for the other resource classes Personnel, PhysicalAsset, and Material [7].

\section{The process model of IEC 62264}

Process segments in IEC 62224 are defined as the smallest elements of manufacturing activities that are visible to business processes. The process model is hierarchically structured, i.e., each process segment can contain further subordinate process segments. The interdependencies between the process segments are realized by references in the form of ProcessSegmentDependencies. These are e.g. AtStart, NotFollow, PossibleParallel and others. All resources like Equipment or Material can be aggregated from process segments as resource specifications via references. The processes in turn can correspond with operation or product definition segments [7].

\section{The transaction model of IEC 62264}

Part 5 of IEC 62264 defines transactions for the exchange of information between applications of business processes and applications of manufacturing processes. The extensive transaction specifications are one of the main reasons for using B2MML as a data model in this concept. The GET/SHOW transaction mechanism is the one that is used in presented concept. The transaction model for GET and SHOW is the PULL transaction model. It is used when a user submits a data request to an information provider [8]. A complete list of the transactions defined in the IEC 62264 is listed Table 1.

Table 1: Transactions in IEC 62264-5 [8]

\begin{tabular}{ll}
\hline Transaction & Transaction model \\
\hline Sync (Change) & PUBLISH \\
Sync (Delete) & PUBLISH \\
Sync (Add) & PUBLISH \\
Get & PULL \\
Show & PULL \\
Confirm, Cancel, & PUSH \\
Acknowledge, Process, & \\
Respond, Change & \\
\hline
\end{tabular}




\section{DEPLOYMENT AND ORCHESTRATION WITH B2MML}

In the present concept B2MML fulfils two functionalities.

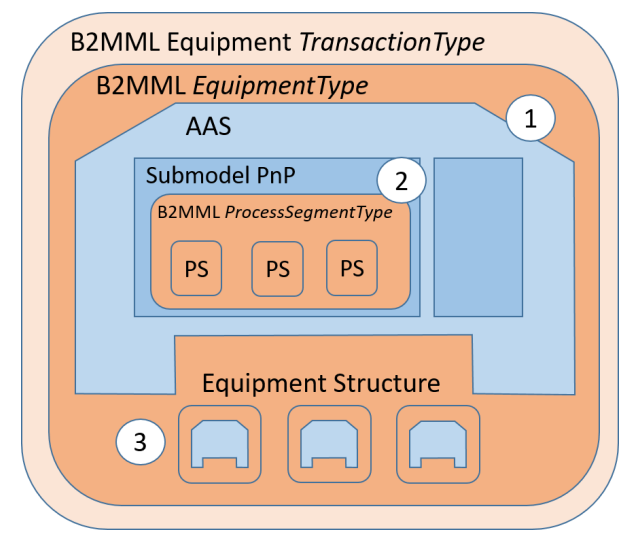

Figure 3: B2MML in combination with AAS

Firstly, it is used as a deployment container for the AAS from the ERP side. The AAS is integrated into the Equipment Type and communicated within the Transaction Types (Fig. 3, (1)).

On the other hand, the associated processes and process chains are modeled in B2MML and integrated into the AAS as submodels for the orchestration of the commissioning (Fig. 3, (2)). This structure can be interconnected at any depth (Fig. 3, 3).

Communication in this concept is realized via Message Queuing Telemetry Transport (MQTT) [3] for deployment of the AAS and with OPC Unified Architecture (OPC UA) [4] as communication protocol between the different AASs in the Plug-and-Produce procedures. Both approaches are explained in more detail below.

\subsection{Deployment}

As described in the introduction, the components of the demonstrator are non-smart components as defined in chapter 2.1 and [11].
When a component is replaced, the corresponding AAS must also be replaced due to the technical conditions in the corresponding hardware and software, in this case the associated RP and the process engine installed on it. For this, an appropriate procedure is necessary to exchange and instantiate the AAS. For a simulated ERP-controlled deployment, the model of the resource type EquipmentType of B2MML (IEC 62264) [6][7] was extended by the model of the administration shell as XML schema. The extension points provided were used for this purpose (Fig. 4). Equivalent to this, the other B2MML resource types (source) PhysicalAsset, Material and Personnel can be extended in the same way.

In this way, the XML representation of the AAS can be integrated unchanged into the data record of an EquipmentType and communicated in a corresponding TransactionType, in this case the ShowEquipmentTransactionType (Fig. 5). The submodel with the B2MML commissioning process queue for the asset is included in the referenced file.

When a new asset is installed, the corresponding administration shell can be read from the equipment data record in parallel, and can be instantiated on the corresponding hardware. To do so, the equipment data, including the administration shell, which is embedded in the transaction types, is transferred using MQTT [3]. The prerequisite for this is that the appropriate software with implemented MQTT is available. In the present approach, this task is performed by the $\mathrm{B} 2 \mathrm{MML}$ process engine.

\subsection{Orchestration}

B2MML is also used to orchestrate the commissioning processes in the submodel "PnP" of the AASs. For this the ProcessSegmentType of B2MML is used (chapter 2.2 [7]). Figure 6 shows such a commissioning sequence from the $\mathrm{X}$-axis of the pneumatic handling system.

The individual commissioning steps are

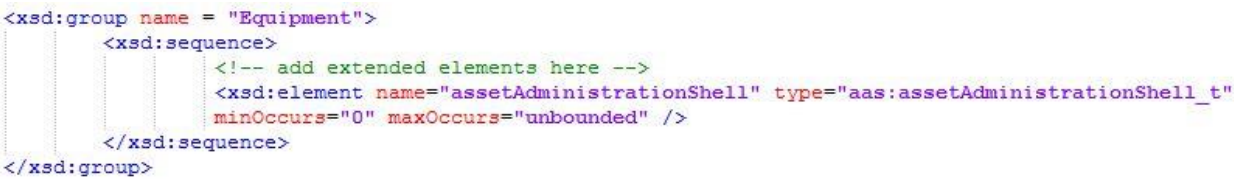

Figure 4: B2MML EquipmentType extension with AAS type 


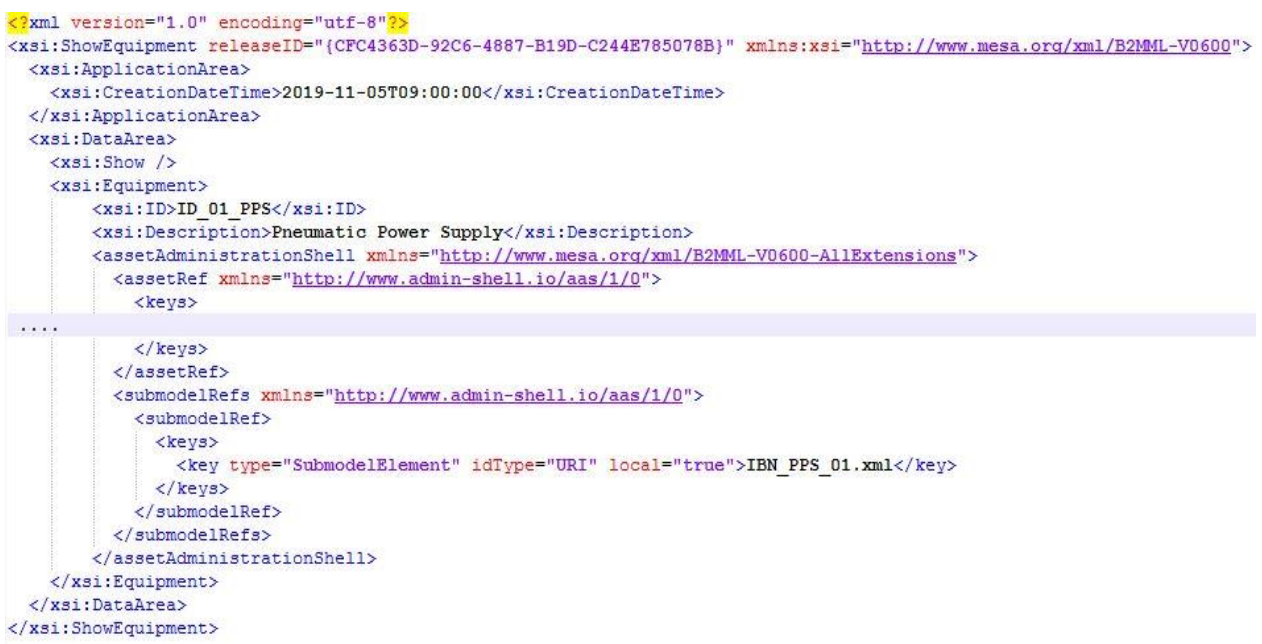

Figure 5: B2MML ShowEquipmentType transaction with integrated AAS

modeled using the ProcessSegmentDependencies of the B2MML process type in accordance with the commissioning requirements for the sequence of the commissioning steps. Since in this concept the dependencies are referenced using the IDs of the processes, the processes must be known among themselves [7]. This is not the case with the commissioning of a complex plant consisting of several components with their different AASs. Therefore the external dependencies are modeled in B2MML parameter types in the form of pre and post conditions, which are assigned to the processes.

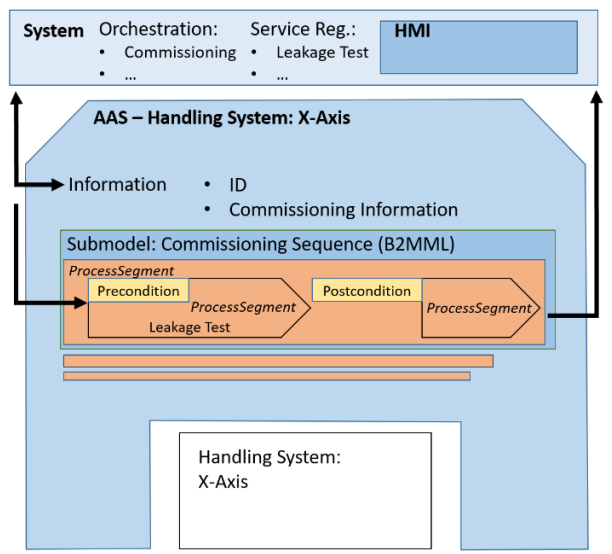

Figure 6: Orchestration of commissioning with B2MML ProcessSegmentType [7]
In order to instantiate and run the defined processes, a process engine for B2MML is required, which must be installed on the respective RPs and PLC. Such a process engine is currently being developed as part of the FL4 project. The (very extensive) details of the process modeling as well as the implementation of the B2MML process engine would exceed the scope of this publication and will therefore be focused separately in a following publication.

\section{TRANSFERABILITY OF THE OVERALL CONCEPT}

The presented concept can be applied with restrictions to the complete life cycle of an administration shell within a company [14] (Fig. 7). The concept is based on non-smart components (chapter 1), which will probably be in operation for several more years. Currently, the concept is applied for type 2 administration shells for fluid power components, but it can also be very useful for assets like cylinders or material, which have typical type 1 administration shells.

\section{Procurement and Inventory}

Once an asset has been acquired, it will first be inventoried. In the present concept, the AAS, which is supplied by the manufacturer, is directly maintained in the system as part of the associated asset in a B2MML type and is therefore directly available at all times. 
Attention has to be paid to ensure that the AAS has to be unpacked before, according to the rules after [2].

\section{Production and Process Planning}

In production and process planning, the added or changed data can be entered natively in the domain specific models. This information can then be used in the AASs. This applies to both, information directly in the AAS, and information regarding communication, topology or orchestrated processes as B2MML in the submodels.

With regard to the submodels, this concept offers the advantage that these can also be integrated natively, provided they are XMLbased, into the B2MML structure [12]. This is especially true for topology information in AutomationML [9], programming information in PLCopen XML [13] or communication specifications for OPC UA [4].

\section{Engineering and Configuration}

In the engineering and configuration phase, the data from the submodels, especially topological planning information and communication (OPC UA), are also available natively, and thus can be read and edited via the corresponding transactions (chapter 2.2, [8]).

\section{Commissioning}

Finally, when the system is put into operation, the AAS, which has been kept up to date by the processes described above, can be instantiated on hardware provided for this purpose. Then the processes, which are also modelled in B2MML, can be executed with the help of a Process Engine. Other information like OPC UA [10] or PLCopen XML can be used for the setup of the asset i.e. its managing hardware and software.

\section{Production and monitoring}

Finally, B2MML can be used in production equivalent to commissioning as a model for automated process chains. For the necessary monitoring, the approach offers the advantage that the AAS, since instantiated from the ERPreadable B2MML data record, is also known from the ERP and thus also for the upper levels of the company hierarchy. Therefore, it can be easier connected and monitored.

\section{CONCLUSION}

Through the combined use of B2MML and Asset Administration Shells it is possible on the one hand to manage and distribute the AASs of the respective assets consistently within an ERP system, or to deploy them. In addition, B2MML, as an implementation of IEC 62264, is very well

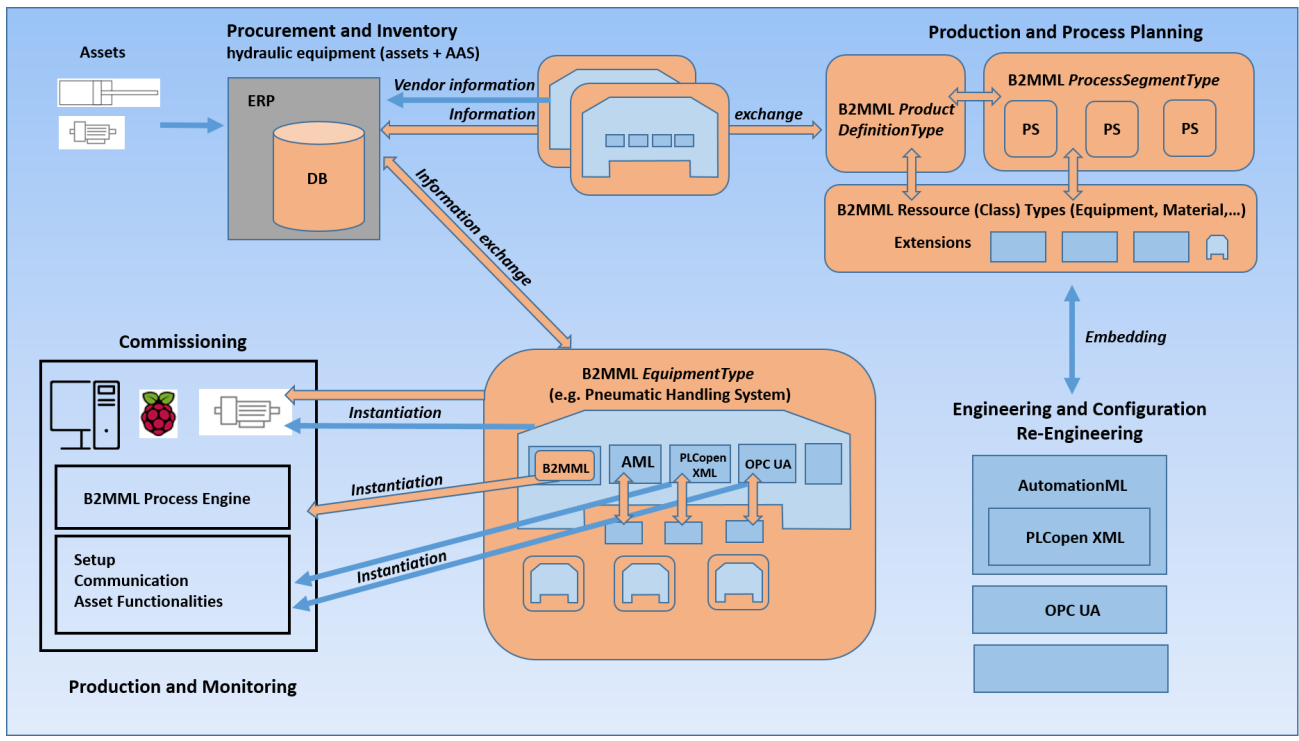

Figure 7: Overall concept of combination of AAS and B2MML 
suited as a submodel for process queues, e.g. for partially and fully automated commissioning. Thus, this concept can support the entire life cycle of an AAS within a company, from the acquisition of the associated asset through installation and commissioning to production and monitoring. The concept was implemented within the project FL4. Of course, further tests and implementations in different contexts are necessary to prove the basic suitability.

\section{ACKNOWLEDGMENTS}

This contribution results from the project 'proofof interoperability of IIoT fluid power components by the example of plug-andproduce', which is funded by the Forschungskuratorium Maschinenbau of the VDMA.

\section{NOMENCLATURE}

$\begin{array}{ll}A A S & \text { Asset Administration Shell } \\ A M L & \text { Automation Mark-up Language } \\ \text { B2MML } & \begin{array}{l}\text { Business to Manufacturing Mark-up } \\ \text { Language }\end{array} \\ \text { CE } & \text { Commissioning Engineer } \\ H M I & \text { Human Machine Interface } \\ I 4.0 & \text { Industry 4.0 } \\ M Q T T & \text { Message Queuing Telemetry Transport } \\ \text { OPC UA } & \text { Open Platform Communications Unified } \\ & \text { Architecture } \\ \text { PLC } & \text { Programmable Logic controller } \\ P n P & \text { Plug-and-Produce } \\ S O A & \text { Service-oriented architecture }\end{array}$

\section{REFERENCES}

[1] VDI / VDE / ZVEI (2016) Fortentwicklung des Referenzmodells für die Industrie 4.0 Komponente Struktur der Verwaltungsschale, Conclusionpaper

[2] VDI / VDE / ZVEI, Details of the Asset Administration Shell, November 2018

[3] http://mqtt.org/

[4] OPC Foundation: OPC-UA, https://opcfoundation.org/about/opctechnologies/opc-ua/, retrieved: November 2019.

[5] International Electrotechnical Commission (IEC). IEC 62264-1:2013 Enterprise-control system integration - Part 1: Models and terminology. International Electrotechnical Commission, Geneva, 2013.
[6] MESA International, Business To Manufacturing Markup Language (B2MML), http://www.mesa.org/en/B2MML.asp, retrieved: November 2019.

[7] International Electrotechnical Commission (IEC), IEC 62264-2:2013 Enterprise-control system integration-Part 2: Objects and attributes for enterprise control system integration, International Standard, Rev. 2.0, June 2013.

[8] International Electrotechnical Commission (IEC), IEC 62264-5:2016 Enterprise-control system integration - Part 5: Business to manufacturing transactions. International Electrotechnical Commission, Geneva, 2016

[9] IEC 62714 - Engineering data exchange format for use in industrial automation systems engineering - AutomationML, www.iec.ch, International Electrotechnical Commission, 2014.

[10] OPC Foundation: ISA-95 for OPC UA. https://opcfoundation.org/marketscollaboration/isa-95/, retrieved: November 2019.

[11] VDI / VDE / ZVEI, Verwaltungsschale in der Praxis, April 2019

[12] Schweizer, H.; Wollschlaeger, M.; Information Model Integration for Service-oriented Manufacturing Operation Management Systems; eKNOW 2019; Athens, February 2019.

[13] PLCopen, TC6-XML Schemes. http://www.plcopen.org/pages/tc6_xml/, retrieved: November 2019.

[14] Wagner, C. et al; The role of the Industry 4.0 Asset Administration Shell and the Digital Twin during the life cycle of a plant; ETFA 2017; Limassol, January 2017. 